\title{
Barriers to Medications for Opioid Use Disorder Among Veterans Involved in the Legal System: a Qualitative Study
}

\author{
Andrea K. Finlay, $P h D^{1,2}$ (D) Erica Morse, $M A^{3}$, Matthew Stimmel, $P h D^{4}$, \\ Emmeline Taylor, BS ${ }^{1,5}$, Christine Timko, $P h D^{1,6}$, Alex H. S. Harris, $P h D^{1,7}$, \\ David Smelson, PsyD ${ }^{8}$, Mengfei Yu, MS ${ }^{7}$, Jessica Blue-Howells, MSW ${ }^{4}$, and \\ Ingrid A. Binswanger, $\mathrm{MD}^{3,9,10}$
}

\begin{abstract}
${ }^{1}$ Center for Innovation to Implementation (Ci2i), VA Palo Alto Health Care System 795 Road (MPD-152), Menlo Park, Willow, CA94025, USA; ${ }^{2}$ Department of Veterans Affairs, National Center on Homelessness Among Veterans 795 Willow Road, Menlo Park 94025, USA; ${ }^{3}$ Institute for Health Research, Kaiser Permanente ColoradoDenver, USA; ${ }^{4}$ Veterans Justice Programs, U.S. Department of Veterans Affairs, Menlo Park, USA; ${ }^{5}$ Department of Clinical Psychology, University of Colorado, Colorado SpringsColorado Springs, USA; 6 Department of Psychiatry and Behavioral Sciences, Stanford University School of MedicineStanford, USA; ' Department of Surgery, Stanford University School of MedicineStanford, USA; ${ }^{8}$ Center for Organization and Implementation Science, Edith Nourse Rogers VA Medical CenterBedford, USA; ${ }^{9}$ Colorado Permanente Medical GroupDenver, USA; ${ }^{10}$ Division of General Internal Medicine, University of Colorado School of MedicineAurora, USA.
\end{abstract}

BACKGROUND: Veterans involved in the legal system are at high risk for overdose but have lower receipt of medications for opioid use disorder than other veterans.

OBJECTIVE: The study aimed to understand barriers to medication access from the perspective of legally involved veterans with opioid use disorder and people who work with these veterans in the Veterans Health Administration (VHA) and the legal system.

DESIGN: This national qualitative study interviewed veterans and stakeholders from 14 geographically diverse VHA facilities to explore perceptions of barriers to medications for opioid use disorder.

PARTICIPANTS: Participants included veterans with a history of opioid use disorder and legal involvement $(n=$ 18), VHA Veterans Justice Programs Specialists $(n=15)$, VHA and community substance use disorder treatment providers $(n=5)$, and criminal justice staff $(n=12)$.

APPROACH: We conducted interviews based on the Consolidated Framework for Implementation Research.

\section{Prior Presentations}

a. Finlay, AK*, Morse, $E^{*}$, Adams, $S^{*}$, Blue-Howells, J*, Timko, C, Harris, AHS, Smelson, D, Stimmel, M, Sardarian, S, Yu, M, \& Binswanger, I (2019, March). Access to pharmacotherapy for opioid use disorder among justiceinvolved veterans: quantitative and qualitative perspectives. Seminar presented at the $12^{\text {th }}$ Academic and Health Policy Conference on Correctional Health, Las Vegas, NV. (*presenters)

b. Finlay, AK, Stimmel, M, \& Blue-Howells, J (2019, July). Decision process impacts on access to pharmacotherapy for opioid use disorder among justice-involved veterans. Invited presentation at the 2019 National Association of Drug Court Professionals featuring Vet Court Con. National Harbor, MD.

c. Finlay, $A K$ (2019, Sept). Decision process impacts on access to pharmacotherapy for opioid use disorder among justice-involved veterans. Invited presentations at the 2019 Veterans Justice Programs Regional Training Conferences, Orlando, FL \& Albuquerque, NM.

Electronic supplementary material The online version of this article (https://doi.org/10.1007/s11606-020-05944-6) contains supplementary material, which is available to authorized users.

Received October 26, 2019

Accepted May 22, 2020

Published online June 24, 2020
Interview transcripts were analyzed using a team-based approach.

KEY RESULTS: Four key barriers, noted by group, were identified: (1) a preference for counseling along with or instead of medications (veterans, Specialists, treatment providers, criminal justice staff); (2) concerns about veterans using medications without a prescription, selling them, or providing them to others (veterans, Specialists, treatment providers, criminal justice staff); (3) concerns about perceived stigma towards medication use (veterans, Specialists, treatment providers, criminal justice staff); and (4) concerns about medication discontinuation after recurrent opioid use (veterans, criminal justice staff). A fifth theme, education, was noted by all stakeholders except providers as important to facilitating use of medications for opioid use disorder. All five themes mapped to the framework construct of knowledge and beliefs about the intervention.

CONCLUSIONS: Based on identified barriers, interventions focused on enhancing medication knowledge, reducing stigma towards use of medications, and increasing knowledge that opioid use may recur during treatment may help increase access to medication for veterans with legal involvement.

KEY WORDS: opioid-related disorders; pharmacotherapy; veterans; criminal justice.

$\mathrm{J}$ Gen Intern Med 35(9):2529-36

DOI: $10.1007 / \mathrm{s} 11606-020-05944-6$

(c) Society of General Internal Medicine (This is a U.S. government work and not under copyright protection in the U.S.; foreign copyright protection may apply) 2020

\section{INTRODUCTION}

Overdose is the leading cause of death among veterans exiting prison. ${ }^{1}$ Medications for opioid use disorder (MOUD) - methadone, buprenorphine, and naltrexone - are effective for reducing mortality and likelihood of repeat 
overdose. ${ }^{2,3}$ Populations involved in the criminal legal system (e.g., in prison, in jail, on parole, on probation, in a specialty court, or formerly incarcerated) also benefit from MOUD, with evidence of reduced opioid use, hospital admissions, incarceration, arrests, and violent and non-violent offenses. ${ }^{4-10}$ Among people exiting prison, MOUD reduces reincarceration risk ${ }^{11}$ and is cost-effective. ${ }^{12}$ Despite evidence on its effectiveness, veterans exiting prison have lower utilization rates of MOUD at Veterans Health Administration (VHA) facilities than veterans not involved in the legal system. ${ }^{13}$

Patient, provider, healthcare system, and legal system barriers may prevent veterans from accessing MOUD. Patients with recent legal involvement may choose not to use MOUD for many reasons, including preferences for reliance on "willpower" to avoid recurrence of use and concerns about "trading one drug for another". ${ }^{14,}{ }^{15}$ Provider barriers to offering MOUD include concerns about non-prescribed use of medications ${ }^{16}$ and numbers of insufficient providers with waivers to prescribe buprenorphine. ${ }^{17}$ Healthcare system barriers include treatment program philosophy (e.g., preferring psychosocial treatments over pharmacotherapy), policy restrictions, and cost concerns. ${ }^{18,19}$ Other barriers include limited provision of MOUD in legal settings ${ }^{20-22}$ and stigma related to legal involvement and opioid use. ${ }^{23}$

In the USA, the VHA is an ideal healthcare system to address barriers to MOUD because it is national and has issued mandates that MOUD be considered for all veterans indicated for treatment. ${ }^{24}$ When released from incarceration, veterans eligible for VHA care can access treatment at more than 1700 VHA locations. There are methadone clinics located at 55 VHA facilities; buprenorphine and naltrexone are provided by all VHA pharmacies; and substance use disorder treatment is offered in more than 220 VHA outpatient and residential treatment programs. Furthermore, the VHA's Veterans Justice Programs have staff, called Specialists, who conduct outreach with veterans in prisons, jails, and courts to connect them with VHA and community care. ${ }^{25}$ Despite mandates and outreach support, in 2012, only $27 \%$ of veterans with a history of prison incarceration received MOUD compared with $33 \%$ of veterans with no known legal involvement. ${ }^{13}$ This study aimed to examine the perspectives of veterans involved in the legal system and those of other stakeholders on potentially modifiable factors that impact access to and receipt of MOUD by veterans involved in the legal system.

\section{METHODS}

\section{Overall Study Design}

We conducted a qualitative study at VHA facilities across the USA. Using quantitative electronic health record data from fiscal years 2016 and 2017 to describe MOUD receipt rates among veterans, ${ }^{26,27} 14$ facilities were selected for qualitative inquiry (Supplementary Appendix). Four types of stakeholders were recruited to capture an array of perspectives: veterans with opioid use disorder with a history of legal involvement, Veterans Justice Programs Specialists, VHA and community substance use disorder treatment providers, and criminal justice staff. The study was approved by the Stanford University Institutional Review Board and the VA Palo Alto Research \& Development committee.

\section{Qualitative Recruitment and Interview Procedure}

Study staff directly contacted Veterans Justice Programs Specialists via email to invite them to participate in interviews. Specialists who agreed to participate were asked to provide study recruitment flyers to potential participants including VHA and community substance use disorder treatment providers, criminal justice staff, and veterans who met eligibility requirements. Specialists also asked potential participants if they could share their contact information with the research team. Distributed flyers were not tracked. Recruitment and interviewing occurred from February 2018 through March 2019.

Participant eligibility requirements were age 18 years or older, English speaking, and able to understand study procedures. Specialists, treatment providers, and criminal justice staff were eligible if in that role at the time of the interview. Veterans were included if they had a history of opioid use or opioid use disorder, within the last 10 years, and a history of legal involvement, defined as having been arrested, in a jail or prison, on probation or parole, or in criminal court within the last 10 years but not incarcerated at the time of the interview. We aimed to include at least one staff stakeholder participant in each group and two veteran participants from each facility.

Telephone interviews were conducted and ranged from 20 to $60 \mathrm{~min}$. Participants consented at the start of the interview. Halfway through each interview, provision of MOUD at their facility was described to the participant. All interviews were audio recorded and transcribed verbatim. A $\$ 30$ check incentive was offered to veterans and non-VHA participants for their participation; most staff declined because of court or grant policies prohibiting receipt of monetary incentives. VHA staff were not offered incentives to participate because of VHA restrictions on research incentives.

\section{Interview Guide}

The Consolidated Framework for Implementation Science (CFIR) model, ${ }^{29}$ which describes various levels of the healthcare system that affect the healthcare program and practice implementation, was used to develop the interview guide. CFIR has been used in previous studies of implementation of substance use disorder treatment in the community ${ }^{30,31}$ and VHA treatment settings. ${ }^{19,} 32$ Questions pertained to CFIR domains of Intervention Characteristics, Outer Setting, Inner Setting, and Characteristics of Individuals. They also included barriers to accessing MOUD, knowledge of and preferences for different MOUD, and philosophy towards addiction treatment. The interview guide was revised after the first five 
interviews to include additional open-ended questions to encourage divergent views.

\section{Analysis}

Interview transcripts were analyzed in ATLAS.ti Version $8 .^{33}$ An a priori codebook was created using the CFIR constructs. Eleven interview transcripts were purposively selected to include participants from each group. Five research team members identified meaningful units of analysis (quotations) in the transcribed interviews. Short phrases of approximately 100 characters or less that summarized the quotation content were created. Short phrases were compared and contrasted with the a priori code list and new codes were generated using a grounded theory approach. ${ }^{34}$ After expanding the a priori codebook using the 11 interviews, the team coded all 11 interviews together and refined the codebook until all important content areas were captured. The remaining transcripts were divided up and coded among four team members. The principal investigator randomly selected a transcript from each team member to verify codes and discuss discrepancies.

Once all the interview transcripts were coded, we extracted the quotations for codes that fit with the purpose of this study. Five research team members read the representative quotes and grouped similar codes together. These code groups were then reviewed and discussed to identify themes. The themes and exemplar quotes were then shared with the broader research team for input, and further refinement was conducted based on their input. For this analysis, themes were limited to those that helped to explain variation in attitudes or beliefs around providing, recommending, or using MOUD among veterans with legal involvement. Although themes in the broader study were mapped to a variety of CFIR constructs, the themes selected for these analyses only mapped to the CFIR domain of Characteristics of Individuals.

\section{RESULTS}

\section{Participant Characteristics}

Across facilities, 16 Veterans Justice Programs Specialists were contacted, 15 of whom participated ( $94 \%$ recruitment response rate). A total of 50 participants completed interviews: 15 Veterans Justice Programs Specialists, 5 treatment providers (4 from VHA, 1 from the community), 12 criminal justice staff, and 18 veterans with a history of legal involvement and opioid use disorder. Treatment providers included 2 medical doctors who prescribed MOUD and 3 masters or doctorate level providers who provided nonpharmacotherapy assessment and treatment. Staff included 2 judges, 8 court staff, and 2 probation officers. Participant demographic characteristics and facility location characteristics are reported in Table 1.
Table 1 Qualitative Interview Participant Demographics and Site Selection Characteristics

\begin{tabular}{ll}
\hline \hline Characteristics & $\boldsymbol{N}(\%)$ or mean (SD) \\
\hline Total sample & $50(100 \%)$ \\
Women & $24(48 \%)$ \\
Age & $43.6(12.2)$ \\
Race & $1(2 \%)$ \\
American Indian & $3(6 \%)$ \\
Asian & $2(4 \%)$ \\
Black & $40(82 \%)$ \\
White & $3(6 \%)$ \\
Hispanic & \\
Highest education level & $8(16 \%)$ \\
High school diploma & $6(12 \%)$ \\
Some college & $8(16 \%)$ \\
Bachelor's degree & $29(39 \%)$ \\
Master's degree & $8(16 \%)$ \\
Doctoral, medical, or professional degree & \\
Performance level of facility by respondent & $12(24 \%)$ \\
High & $15(30 \%)$ \\
Low & $5(10 \%)$ \\
Increasing & $18(36 \%)$ \\
Decreasing & \\
Geographic location of facility by respondent & $6(12 \%)$ \\
Northeast & $9(18 \%)$ \\
South & $12(24 \%)$ \\
Midwest & $23(46 \%)$ \\
West &
\end{tabular}

SD standard deviation

\section{Identified Themes}

Four interrelated barriers to MOUD access for veterans with legal involvement were identified, including ${ }^{1}$ a preference for counseling along with or instead of medications ${ }^{2}$; concerns about veterans using medications without a prescription, selling them, or providing them to others ${ }^{3}$; concerns about perceived stigma towards medication use; and ${ }^{4}$ concerns about medication discontinuation after recurrent opioid use. A fifth theme that was both a barrier and a facilitator was identified ${ }^{5}$ : knowledge and education about medications. All themes are mapped to the CFIR construct of Knowledge and Beliefs about the Intervention in the Characteristics of Individuals domain, which is the attitudes, beliefs, and knowledge about the intervention. ${ }^{29}$ The barriers are presented below separately by group type.

Veteran Perspectives. Counseling. Veterans described the importance of counseling instead of or in addition to medications. Some veterans preferred psychosocial treatments because of prior positive or negative experiences with MOUD. For example, one veteran explained changing his preference from medications to psychosocial treatment:

If you would have asked me back in the day, I would've told you Suboxone, but I think therapy is probably the best thing. You have to get to the root of an issue... You know, if you just trim branches on a tree, they're going to grow back. You've got to dig up the root to get that [issue] away. Veteran 
Counseling for opioid use disorder was not always distinguished from counseling for co-occurring mental health disorders. Some veterans attributed opioid use disorder to mental health issues, especially post-traumatic stress disorder (PTSD), and expressed beliefs that counseling for mental health issues was needed to address opioid use disorder. For example, a male veteran prescribed suboxone explained:

I think that in what I've gone through so far, that PTSD has a lot to do with the opiate use and drug use. And I think if they would work on that a lot more, it would help a person out. Veteran

Non-Prescribed Medication Use. Some veterans mentioned using or observing others using medications in non-prescribed ways, such as buying or selling medications. One veteran explained that he perceived that criminal justice agencies were unwilling to provide MOUD because of concerns around nonprescribed use:

Ninety percent of the time, their [jail staff] mentality is if somebody is trying to get [medications] for somebody who's dealing with opioid dependency, they're probably going to use it in a negative way. Whether it's selling it or abusing it or saving it and taking a lot at one time. Veteran

Stigma. Veterans expressed experiences of stigma because of their MOUD use. One veteran said he did not "advertise" his MOUD use to avoid stigma. Other veterans expressed negative attitudes towards MOUD:

I don't care what anybody has to say, suboxone gets you high. It will to a certain amount get you high. And methadone, for a fact gets you stoned because it's still a narcotic. Veteran

However, some veterans expressed positive attitudes towards MOUD, including that it helped them address their opioid use disorder.

Recurrent Opioid Use. Veterans expressed concerns that their providers would discontinue their MOUD after recurrent opioid use following abstinence. One veteran explained that he was immediately titrated off his medications when his urinalysis test was positive for opioids, which caused him to stop attending treatment visits. Another veteran explained:
Sometimes I think that they [treatment providers] shouldn't be so hard on you if you have a relapse or something like that... They threaten to kick you off [of your medications]. Veteran

Education. A lack of education was described as a barrier to MOUD, but veterans described learning about MOUD from VHA classes, from one-on-one conversations with treatment providers, and from other veterans. One veteran explained:

I go to class three times a week, three hours a night. And study about PTSD and drug addiction...It's been very helpful. Veteran

Veterans Justice Programs Specialist Perspectives. Counseling. Although Specialists were in favor of MOUD, a combination of treatments, such as individual therapy and residential treatment, was viewed as the most effective in treating opioid use disorder.

Non-Prescribed Medication Use. Specialists described that non-prescribed use of medications was an issue, such as veterans selling medications for income because they were unable to obtain employment due to their legal history. Specialists observed that previous experiences with people who sold or used medications in non-prescribed ways created concerns among criminal justice partners about allowing access to MOUD. Other Specialists noted instances where the jails or prisons they worked with did not allow medications because of concerns about "diversion" to other incarcerated people.

Stigma. Specialists perceived stigma to be present among veterans, providers, and criminal justice staff members, which created a barrier to allowing veterans to use MOUD:

There is a stigma attached, sometimes, by the attorneys or some people in the justice system when they hear that the VA or a treatment provider are recommending that they [veterans] go on some type of medication. Specialist

Attitudes expressed included a belief that medications are "substituting one addiction for another" and that patients should be able to resolve their opioid use disorder without medications. Sometimes, the perceived stigma was explained to be related to an abstinence-only model of treatment that considers medication use as not abstinent, such as 12-step groups.

Recurrent Opioid Use. This theme was not expressed by Veterans Justice Programs Specialists. 
Education. Respondents described a limited or incorrect knowledge of substance use disorders and evidence-based treatments, such as MOUD, as a barrier to treatment. Other respondents described how they overcame their own stigma towards MOUD or lack of knowledge through education at conferences and online trainings:

I've learned...more about the brain of someone who's addicted and how they can't often times make the right choices for themselves when they're having intense cravings... it's really not a substituting one addiction for another. Specialist

Substance Use Disorder Treatment Provider Perspectives. Counseling. Providers noted that among staff in many treatment programs there was an "old school" model of treatment. These treatment models, such as 12-step groups, focused on abstinence-only and that it is not appropriate to use MOUD with the implied or stated perception that abstinenceonly models consider abstinence to be no medication use.

Non-Prescribed Medication Use. Providers noted that concerns that patients would use medications in nonprescribed ways, such as sharing with other people, factored into decision-making about prescribing. One provider explained that methadone was the most common medication that was prescribed to veterans involved in the legal system with opioid use disorder because of concerns about "diversion."

Stigma. Providers described that veterans reporting feeling stigma towards MOUD and did not want to use MOUD because their 12-step group members did not support it, but no providers described any stigma towards MOUD. One provider described a veteran who preferred suboxone to methadone so they did not have to be seen going to the methadone clinic. A provider noted that family members who share negative beliefs about medications influenced veterans' use of this treatment modality:

We've had so many guys say, "I relapsed." "Well what happened?" "Well my wife or my girlfriend, my mom, my dad ... they didn't like the idea of me being on methadone or Suboxone. They said it didn't mean I was actually clean." So there's definitely a lot of stigma around it. Provider

Recurrent Opioid Use. This theme was not a barrier among providers.

Education. This theme was not a barrier or facilitator among providers.
Criminal Justice Staff Perspectives. Counseling. Among staff, there was a clear preference for counseling along with or instead of MOUD. One court discontinued allowing the use of methadone because it was not offered in conjunction with counseling. In some cases, criminal justice respondents had a prior substance use disorder themselves and found mutualhelp groups or psychosocial treatments to be beneficial. Others explained that mental health issues, especially PTSD, needed to be resolved in counseling to address the veteran's opioid use. Some respondents expressed concern about the length of time a veteran may need to be on medication and viewed it as a short-term intervention:

You can't be on Suboxone for the rest of your life, or you can't be Vivitrol for the rest of your life. You've got learn how to deal with your triggers and get your safeguards. I think throwing the cognitive behavioral stuff in with that is more important for the long run of the client. Criminal Justice Staff

Non-Prescribed Medication Use. Criminal justice staff mentioned concerns about patients selling medications or non-prescribed use. They said jails and prisons were not going to prescribe or provide MOUD because of concerns it would be sold. One court staff member said:

My belief now, because of Vivitrol has actually improved whereas before, when we had so many people on methadone and Suboxone, they abused it. It was very difficult to have someone that didn't abuse there Suboxone in some way. Whether they used too much of it and then ran out and so then they would resort to getting stuff illegally. Criminal Justice Staff

Stigma. Some criminal justice participants reported a perceived stigma towards prescribing and using MOUD, especially methadone:

There's still a stigma around methadone... A long line of strung-out people lumped against the side of a building waiting for the methadone clinic to open. There's still that kind of stigma Criminal Justice Staff

They suggested that the stigma around MOUD was partially caused by a lack of understanding about the efficacy and safety of medications when used as prescribed.

Recurrent Opioid Use. Some respondents perceived that criminal justice staff and treatment programs preferred 
responding to recurrent opioid use in a punitive manner that limited access to MOUD. For example, a veteran who used opioids would be sent to jail or asked to leave a treatment program. However, other criminal justice staff reported that punitive responses were not always used.

Education. Respondents discussed that educational programs were helpful in understanding how MOUD was of benefit to patients with opioid use disorder. One criminal justice staff member noted that a yearly conference their court team attended provided training on MOUD.

\section{DISCUSSION}

Despite a substantial public health focus on improving access to and quality of care to address the opioid epidemic and mandates within the VHA to provide MOUD, our results suggest four major barriers to medication use for veterans involved in the legal system with opioid use disorder. A preference for counseling may stem from a great familiarity with counseling than MOUD or a belief that MOUD is not effective. ${ }^{35}$ Restricting access to MOUD after recurrent opioid use may indicate a lack of knowledge that restricting medications may exacerbate recurrent use and overdose. Some of the stigma towards MOUD may be due to limited understanding of the efficacy and safety of MOUD ${ }^{36}$ and that legal involvement is common for people with opioid use disorder. ${ }^{37}$ Education for patients, providers, and criminal justice staff was highlighted as an approach to overcome these barriers.

\section{Educational Programming}

Stakeholders described classes, conferences, and online training that educated them on the benefits of MOUD. Expanding educational offerings may address some of the beliefs and attitudes about preferences for counseling and responses to recurrent opioid use. These results reflect the CFIR construct of Knowledge and Beliefs about the Intervention, which states that knowledge of an intervention and its effectiveness is required for skillful implementation. ${ }^{29}$ The majority of published research and clinical guidelines indicate that MOUD alone $^{38,39}$ or in combination with psychosocial treatment ${ }^{40,41}$ is the strongest evidence-based treatment for opioid use disorder. An intervention program called Medication First, modeled after Housing First principles, emphasizes access to MOUD without requiring psychosocial services or discontinuation for non-adherence ${ }^{42}$ and has preliminary evidence of effectiveness. ${ }^{43}$ Housing First models are common in $\mathrm{VHA}^{44}$; thus, Medication First may be adopted more readily because this healthcare system is familiar with the underlying principles. For patients involved in the legal system with cooccurring disorders, wraparound treatment is effective and recommended, ${ }^{45,46}$ but evidence supporting psychosocial treatments for opioid use disorder is mixed. ${ }^{41,47}$
Developing an understanding that recurrent opioid use is common when working with patients with opioid use disorder is needed for providers and criminal justice partners. Although more than two-thirds of patients who received methadone or buprenorphine experienced recurrent opioid use within 5 years, ${ }^{48}$ the risk of recurrent use is reduced when receiving opioid agonist treatment compared with that receiving psychosocial treatment alone. ${ }^{49}$

Patients, family members, and clinic staff may have stigma towards MOUD, ${ }^{50-53}$ and for providers, this stigma may impact their subsequent decisions to prescribe these medications. Concerns may be that a veteran will sell or use medications in a non-prescribed way. The weight these concerns are given in deciding whether to allow or prescribe medications may be explained by the availability heuristic - a cognitive process in which events that are easier to call to mind are considered to have a higher likelihood. ${ }^{54}$ For example, if a court participant is recently found to be selling his medications, this one experience may outweigh the many other court participants who are using MOUD as prescribed. When medications are used in non-prescribed ways, it is often in the context of treatment - to help others in withdrawal or to prevent recurrence of heroin use - than for euphoric purposes ${ }^{55-58}$ —education efforts around this topic may reduce concerns. A shortage of available MOUD treatment may explain non-prescribed use or sharing of medications, ${ }^{59,}{ }^{60}$ suggesting that more work is needed to understand and address the treatment needs of people with opioid use disorder to reduce non-prescribed use of MOUD. Strict tracking and testing requirements can also be helpful to mitigate concerns about sharing or selling of medications without limit provision of treatment. ${ }^{46,51}$ Congruent with the CFIR construct of Knowledge and Beliefs about the Intervention, these negative beliefs about the intervention or behaviors related to the intervention may create passive or active resistance. Drawing parallels between MOUD and other chronic conditions that use medications long-term may change attitudes towards these medications. For example, among veterans with co-occurring schizophrenia, bipolar disorder, posttraumatic stress or major depressive disorder, and alcohol use disorder, 69 to $82 \%$ received medications for their mental health condition, ${ }^{61}$ indicating their comfort and willingness to try medications for mental health conditions.

\section{Limitations}

Despite the strengths of this study, which include geographic and stakeholder diversity and a focus on VHA which has not been examined before, there are a few limitations. First, we were unable to recruit criminal justice partners who work in jails or prisons and recruited only a small number of substance use disorder providers. Through purposive sampling, we were able to achieve more than 9 interviews in three of the stakeholder groups, which is a reasonable approximation for code saturation; however, 16 to 24 interviews are considered a reasonable approximation for meaning saturation. ${ }^{28}$ 
Additional interviews with the Specialist, criminal justice staff, and substance use disorder provider groups may have provided further insights or dimensions. Second, we did not interview any veterans who did not use or were ineligible for VHA healthcare. The MOUD options for veterans not in the VHA system may vary, which may influence perceptions of these medications. Finally, we focused on only attitudes and beliefs in this study, which limited our results to only one aspect of the healthcare system - captured by the CFIR construct of Knowledge and Beliefs about the Intervention. However, there were other barriers to and facilitators of MOUD expressed by participants that we plan to explore in future studies.

\section{CONCLUSIONS}

Veterans involved in the legal system with opioid use disorder experience various barriers to receipt of MOUD that stem from attitudes and beliefs about the medications. Testing existing intervention programs used with other populations with opioid use disorder, such as Medication First, that can be delivered in VHA and criminal justice settings will be important next steps for future research. Interventions that reduce stigmatization of MOUD and address knowledge gaps of veterans, providers, and criminal justice staff are needed to improve access and utilization of MOUD.

Acknowledgments: We would like to thank all stakeholders for participating in this study.

Corresponding Author: Andrea K. Finlay, PhD; Center for Innovation to Implementation (Ci2i), VA Palo Alto Health Care System 795 Road (MPD-152), Menlo Park, Willow, CA 94025, USA (e-mail: Andrea Finlay@va.gov).

Funding Information Research reported in this publication was supported by the National Institute on Drug Abuse of the National Institutes of Health under Award Number R21 DA041489. Dr. Finlay was supported by a Department of Veterans Affairs Health Services Research \& Development (VA HSR\&D) Career Development Award (CDA 13-279). Dr. Timko was supported by a VA HSR\&D Senior Research Career Scientist award (RCS 00-001). Dr. Harris was funded as a VA HSR\&D Research Career Scientist (RCS 14-232).

\section{Compliance with Ethical Standards:}

The study was approved by the Stanford University Institutional Review Board and the VA Palo Alto Research \& Development committee.

Conflict of Interest: The authors declare that they do not have a conflict of interest.

Disclaimer: The content is solely the responsibility of the authors and does not necessarily represent the official views of the National Institutes of Health. The views expressed in this article are those of the authors and do not necessarily reflect the position nor policy of the Department of Veterans Affairs (VA) or the US government. The VA had no role in the study design; in the collection, analysis, and interpretation of data; in the writing of the report; or in the decision to submit the paper for publication.

\section{REFERENCES}

1. Wortzel HS, Blatchford P, Conner L, Adler LE, Binswanger IA. Risk of death for veterans on release from prison. J Am Acad Psychiatry Law. 2012;40(3):348-54.

2. Sordo L, Barrio G, Bravo MJ, Indave BI, Degenhardt L, Wiessing L, et al. Mortality risk during and after opioid substitution treatment: systematic review and meta-analysis of cohort studies. BMJ. 2017;357:j1550.

3. Larochelle MR, Bernson D, Land T, Stopka TJ, Wang N, Xuan Z, et al. Medication for opioid use disorder after nonfatal opioid overdose and association with mortality: a cohort study. Ann Intern Med. 2018 Aug 7;169(3): 137-45.

4. Coviello DM, Cornish JW, Lynch KG, Boney TY, Clark CA, Lee JD, et al. A multisite pilot study of extended-release injectable naltrexone treatment for previously opioid-dependent parolees and probationers. Subst Abus. 2012;33(1):48-59.

5. Dolan KA, Shearer J, White B, Zhou J, Kaldor J, Wodak AD. Four-year follow-up of imprisoned male heroin users and methadone treatment: mortality, re-incarceration and hepatitis $\mathrm{C}$ infection. Addiction. 2005;100(6):820-8.

6. Gryczynski J, Kinlock TW, Kelly SM, O'Grady KE, Gordon MS, Schwartz RP. Opioid agonist maintenance for probationers: patientlevel predictors of treatment retention, drug use, and crime. Subst Abus. 2012;33(1):30-9.

7. Kelly SM, O'Grady K E, Jaffe JH, Gandhi D, Schwartz RP. Improvements in outcomes in methadone patients on probation/parole regardless of counseling early in treatment. J Addict Med. 2013;7(2):133-8.

8. Russolillo A, Moniruzzaman A, McCandless LC, Patterson M, Somers JM. Associations between methadone maintenance treatment and crime: a 17-year longitudinal cohort study of Canadian provincial offenders. Addiction. 2018 Apr;113(4):656-67.

9. Russolillo A, Moniruzzaman A, Somers JM. Association of methadone treatment with substance-related hospital admissions among a population in Canada with a history of criminal convictions. JAMA Netw Open. 2019;2(3):e190595.

10. Evans EA, Zhu Y, Yoo C, Huang D, Hser YI. Criminal justice outcomes over 5 years after randomization to buprenorphine-naloxone or methadone treatment for opioid use disorder. Addiction. 2019;114(8):1396-404

11. Larney S, Toson B, Burns L, Dolan $\mathbf{K}$. Effect of prison-based opioid substitution treatment and post-release retention in treatment on risk of re-incarceration. Addiction. 2012;107(2):372-80.

12. Gisev N, Shanahan M, Weatherburn DJ, Mattick RP, Larney S, Burns $\mathbf{L}$, et al. A cost-effectiveness analysis of opioid substitution therapy upon prison release in reducing mortality among people with a history of opioid dependence. Addiction. 2015;110(12):1975-84.

13. Finlay AK, Harris AH, Rosenthal J, Blue-Howells J, Clark S, McGuire $\mathbf{J}$, et al. Receipt of pharmacotherapy for opioid use disorder by justiceinvolved U.S. Veterans Health Administration patients. Drug Alcohol Depend. 2016;160:222-6.

14. Fox AD, Maradiaga J, Weiss L, Sanchez J, Starrels JL, Cunningham Co. Release from incarceration, relapse to opioid use and the potential for buprenorphine maintenance treatment: a qualitative study of the perceptions of former inmates with opioid use disorder. Addict Sci Clin Pract. 2015; 10(1):2.

15. Larney $\mathbf{S}$, Zador $\mathbf{D}$, Sindicich $\mathbf{N}$, Dolan $\mathbf{K}$. A qualitative study of reasons for seeking and ceasing opioid substitution treatment in prisons in New South Wales, Australia. Drug Alcohol Rev. 2017;36(3):305-10.

16. Yokell MA, Zaller ND, Green TC, Rich JD. Buprenorphine and buprenorphine/naloxone diversion, misuse, and illicit use: an international review. Curr Drug Abuse Rev. 2011;4(1):28-41.

17. Rosenblatt RA, Andrilla CH, Catlin M, Larson EH. Geographic and specialty distribution of US physicians trained to treat opioid use disorder. Ann Fam Med. 2015;13(1):23-6.

18. Oliva EM, Maisel NC, Gordon AJ, Harris AH. Barriers to use of pharmacotherapy for addiction disorders and how to overcome them. Curr Psychiatry Rep. 2011;13(5):374-81.

19. Finlay AK, Wong JJ, Ellerbe LS, Rubinsky A, Gupta S, Bowe TR, et al. Barriers and facilitators to implementation of pharmacotherapy for opioid use disorders in VHA residential treatment programs. J Stud Alcohol Drugs. 2018;79(6):909-17.

20. Friedmann PD, Hoskinson R, Gordon M, Schwartz R, Kinlock T, Knight $\mathbf{K}$, et al. Medication-assisted treatment in criminal justice agencies affiliated with the criminal justice-drug abuse treatment studies (CJ-DATS): availability, barriers, and intentions. Subst Abus. 2012;33(1):9-18. 
21. Matusow H, Dickman SL, Rich JD, Fong C, Dumont DM, Hardin C, et al. Medication assisted treatment in US drug courts: results from a nationwide survey of availability, barriers and attitudes. J Subst Abuse Treat. 2013;44(5):473-80.

22. Reichert J, Gleicher L. Probation clients' barriers to access and use of opioid use disorder medications. Health Justice. 2019;7(1):10.

23. McElrath K. Medication-assisted treatment for opioid addiction in the United States: Critique and commentary. Subst Use Misuse. 2018;53(2):334-43.

24. Department of Veterans Affairs. Uniform Mental Health Services in VA medical centers and clinics. VHA Handbook 1160.1. Washington, DC: US Department of Veterans Affairs, Veterans Health Administration; 2008.

25. Blue-Howells JH, Clark SC, van den Berk-Clark C, McGuire JF. The U.S. Department of Veterans Affairs Veterans Justice Programs and the sequential intercept model: case examples in national dissemination of intervention for justice-involved veterans. Psychol Serv. 2013;10(1):4853. doi:https://doi.org/10.1037/a0029652

26. Finlay AK, Binswanger IA, Timko C, Smelson D, Stimmel MA, Yu M, et al. Facility-level changes in receipt of pharmacotherapy for opioid use disorder: Implications for implementation science. J Subst Abuse Treat. 2018 Dec;95:43-7.

27. Finlay AK, Harris AHS, Timko C, Yu M, Smelson D, Stimmel M, et al. Disparities in access to medications for opioid use disorder in the Veterans Health Administration. Manuscript submitted for publication. 2019

28. Hennink MM, Kaiser BN, Marconi VC. Code saturation versus meaning saturation: how many interviews are enough? Qual Health Res. 2017 Mar;27(4):591-608.

29. Damschroder LJ, Aron DC, Keith RE, Kirsh SR, Alexander JA, Lowery JC. Fostering implementation of health services research findings into practice: a consolidated framework for advancing implementation science. Implementation Sci. 2009;4:50.

30. Manuel JK, Hagedorn HJ, Finney JW. Implementing evidence-based psychosocial treatment in specialty substance use disorder care. Psychol Addict Behav. 2011;25(2):225-37.

31. Sorensen JL, Kosten T. Developing the tools of implementation science in substance use disorders treatment: applications of the Consolidated Framework for Implementation Research. Psychol Addict Behav. 2011;25(2):262-8.

32. Finlay AK, Ellerbe LS, Wong JJ, Timko C, Rubinsky AD, Gupta S, et al. Barriers to and facilitators of pharmacotherapy for alcohol use disorder in VA residential treatment programs. J Subst Abuse Treat. 2017;77:38-43.

33. Atlas.ti Scientific Software Development $\mathrm{GmbH}$. Atlas.ti Version 8 [Computer Software]. Berlin, Germany: Scientific Software Development; 2019.

34. Bryant A, Charmaz K, editors. The Sage Handbook of Grounded Theory Newbury Park, CA: Sage Publications; 2010.

35. Huhn AS, Tompkins DA, Dunn KE. The relationship between treatment accessibility and preference amongst out-of-treatment individuals who engage in non-medical prescription opioid use. Drug Alcohol Depend. 2017 Nov 1;180:279-85.

36. Uebelacker LA, Bailey G, Herman D, Anderson B, Stein M. Patients' beliefs about medications are associated with stated preference for methadone, buprenorphine, naltrexone, or no medication-assisted therapy following inpatient opioid detoxification. J Subst Abuse Treat. 2016 Jul;66:48-53.

37. Winkelman TNA, Chang Vw, Binswanger IA. Health, polysubstance use, and criminal justice involvement among adults with varying levels of opioid use. JAMA Netw Open. 2018;1(3):e180558.

38. Department of Veterans Affairs \& Department of Defense. VA/DoD clinical practice guideline for the management of substance use disorders. Washington, DC: Authors; 2015.

39. Amato L, Minozzi S, Davoli M, Vecchi S. Psychosocial combined with agonist maintenance treatments versus agonist maintenance treatments alone for treatment of opioid dependence. Cochrane Database Syst Rev. 2011 (10):CD004147.

40. Kampman K, Jarvis M. American Society of Addiction Medicine (ASAM) national practice guideline for the use of medications in the treatment of addiction involving opioid use. J Addict Med. 2015;9(5):358-67.

41. Dugosh K, Abraham A, Seymour B, McLoyd K, Chalk M, Festinger D. A systematic review on the use of psychosocial interventions in conjunction with medications for the treatment of opioid addiction. J Addict Med. 2016;10(2):93-103.
42. Winograd RP, Presnall N, Stringfellow E, Wood C, Horn P, Duello A. et al. The case for a medication first approach to the treatment of opioid use disorder. Am J Drug Alcohol Abuse. 2019;45(4):333-40.

43. Winograd RP, Wood CA, Stringfellow EJ, Presnall N, Duello A, Horn P, et al. Implementation and evaluation of Missouri's Medication First treatment approach for opioid use disorder in publicly-funded substance use treatment programs. J Subst Abuse Treat. 2019 Jun 26.

44. Montgomery AE, Hill L, Culhane DP, Kane V. Housing First implementation brief. Washington, DC: VA National Center on Homelessness Among Veterans, U.S. Department of Veterans Affairs, 2014.

45. Smelson D, Farquhar I, Fisher W, Pressman K, Pinals DA, Samek B, et al. Integrating a co-occurring disorders intervention in drug courts: an open pilot trial. Community Ment Health J. 2019;55(2):222-31.

46. Westreich LM. Use of medication-assisted treatment in the justice system: a medical perspective. Journal for Advancing Justice. 2019;2:519.

47. Connery HS. Medication-assisted treatment of opioid use disorder: review of the evidence and future directions. Harv Rev Psychiatry. 2015;23(2):63-75.

48. Zhu Y, Evans EA, Mooney LJ, Saxon AJ, Kelleghan A, Yoo C, et al. Correlates of long-term opioid abstinence after randomization to methadone versus buprenorphine/naloxone in a multi-site trial. J Neuroimmune Pharmacol. 2018;13(4):488-97.

49. Clark RE, Baxter JD, Aweh G, O'Connell E, Fisher WH, Barton BA. Risk factors for relapse and higher costs among Medicaid members with opioid dependence or abuse: opioid agonists, comorbidities, and treatment history. J Subst Abuse Treat. 2015;57:75-80.

50. Muthulingam D, Bia J, Madden LM, Farnum SO, Barry DT, Altice FL. Using nominal group technique to identify barriers, facilitators, and preferences among patients seeking treatment for opioid use disorder: A needs assessment for decision making support. J Subst Abuse Treat. 2019; 100:18-28.

51. Andrilla CHA, Moore TE, Patterson DG. Overcoming barriers to prescribing buprenorphine for the treatment of opioid use disorder: recommendations from rural physicians. J Rural Health. 2019;35(1):113-21.

52. Gallagher JR, Marlowe DB, Minasian RM. Participant perspectives on medication-assisted treatment for opioid use disorders in drug court. Journal for Advancing Justice. 2019;2:39-54.

53. Dorman A. Badeti J, Boros A. An exploration of knowledge, opinions, and stigma regarding medication-assisted treatment among treatment and criminal justice professionals. Journal for Advancing Justice. 2019;2:93-108.

54. Dineen KK. Addressing prescription opioid abuse concerns in context: synchronizing policy solutions to multiple complex public health problems. Law \& Psychol Rev. 2016;40:1.

55. Johnson B, Richert T. Non-prescribed use of methadone and buprenorphine prior to opioid substitution treatment: lifetime prevalence, motives, and drug sources among people with opioid dependence in five Swedish cities. Harm Reduct J. 2019;16(1):31.

56. Havnes IA, Clausen T, Middelthon AL. 'Diversion' of methadone or buprenorphine: 'harm' versus 'helping'. Harm Reduct J. 2013;10:24.

57. Harris $\mathbf{M}$, Rhodes $\mathbf{T}$. Methadone diversion as a protective strategy: the harm reduction potential of 'generous constraints'. Int J Drug Policy. 2013;24(6):e43-50.

58. Larance B, Lintzeris $\mathbf{N}$, Ali R, Dietze $\mathbf{P}$, Mattick R, Jenkinson R, et al. The diversion and injection of a buprenorphine-naloxone soluble film formulation. Drug Alcohol Depend. 2014;136:21-7.

59. Johnson B, Richert T. Diversion of methadone and buprenorphine from opioid substitution treatment: a staff perspective. J Psychoactive Drugs. 2014;46(5):427-35

60. Richert T, Johnson B. Long-term self-treatment with methadone or buprenorphine as a response to barriers to opioid substitution treatment: the case of Sweden. Harm Reduct J. 2015;12:1.

61. Rubinsky AD, Chen C, Batki SL, Williams EC, Harris AH. Comparative utilization of pharmacotherapy for alcohol use disorder and other psychiatric disorders among U.S. Veterans Health Administration patients with dual diagnoses. J Psychiatr Res. 2015;69:150-7.

Publisher's Note: Springer Nature remains neutral with regard to jurisdictional claims in published maps and institutional affiliations. 